\title{
Analyse de la structure et du fonctionnement de la filière fourrage dans les villes de Ouagadougou et Bobo-Dioulasso au Burkina Faso
}

\author{
H.O. SANON* ${ }^{*}$ S. SOME/SANOU, M.F. OBULBIGA, F.A. OUBDA et I. BAMOUNI \\ Institut de l'Environnement et de Recherches Agricoles; Centre de Recherche Environnementale Agricole et de \\ Formation de Kamboinsé ; Département Productions Animales. 01 B.P. 476 Ouagadougou, Burkina Faso. \\ *Auteur correspondant; E-mail: hadja_osanon@yahoo.fr; tel. (226) 70102310
}

\section{REMERCIEMENTS}

Les travaux dans cet article rentrent dans le cadre du Projet de recherche intitulé "Accroitre la rentabilité financière des cultures fourragères", financé par le Fonds Compétitif National (FCN) du Programme de Productivité Agricole en Afrique de l'Ouest (PPAAO), a qui les auteurs adressent leurs vifs remerciements.

\section{RESUME}

Cette étude a été conduite dans les deux grandes villes du Burkina Faso (Ouagadougou et BoboDioulasso), dans le but d'analyser la structure et le fonctionnement de l'activité de commercialisation du fourrage qui est en pleine expansion, en vue de proposer des actions d'amélioration et de sauvegarde de l'environnement. Elle a consisté dans un premier temps à faire un inventaire des sites de vente de fourrage, puis à conduire des enquêtes auprès des vendeurs de fourrage et accessoirement auprès des éleveurs acheteurs de fourrage. Douze (12) sites ont été recensés dans la ville de Ouagadougou et 15 sites dans la ville de BoboDioulasso. La date d'implantation des sites est variable, les plus anciens ont 27 ans et 30 ans respectivement à Ouagadougou et à Bobo-Dioulasso. Les fourrages vendus sont de divers types : le fourrage vert, le foin, la paille de céréale, les fanes de légumineuse et des fourrages ligneux. Ces fourrages sont collectés sans aucune compensation, pouvant compromettre la productivité de ces sites. Les coûts sont plus élevés pour les fanes de légumineuses (200-400 F CFA/kg) et le fourrage vert (50-82 F CFA/kg) dans les deux villes. L'activité est jugée rentable, permettant d'engranger des revenus de 67166 F CFA et 26335 F CFA par mois respectivement à Ouagadougou et à Bobo-Dioulasso. Les fourrages achetés sont destinés prioritairement à l'alimentation des ovins. Une meilleure organisation des acteurs s'impose pour la pérennité de l'activité et la sauvegarde de l'environnement.

(C) 2018 International Formulae Group. All rights reserved.

Mots clés : Commercialisation, élevage urbain, fanes, fourrage, pailles

\section{Analysis of the structure and operation of the forage sector in the cities of Ouagadougou and Bobo-Dioulasso}

\begin{abstract}
This study was conducted in Burkina Faso's two major cities (Ouagadougou and Bobo-Dioulasso), with the aim to analyse the structure and operation of the expanding forage marketing business, with a view to propose actions for improvement and environmental protection. It consisted first in making an inventory of the
\end{abstract}


fodder selling sites, then to make surveys with the fodder sellers and accessorily with the livestock owners purchasing fodder. Twelve (12) sites were identified in the city of Ouagadougou and 15 sites in the city of Bobo-Dioulasso. The date of implantation of the sites is variable, the oldest are 27 years and 30 years respectively in Ouagadougou and Bobo-Dioulasso. Fodder sold are of various types: green fodder, hay, cereal straw, legume haulms and browse fodder. These fodder are collected without any compensation, which can compromise the productivity of these sites. The costs are higher for the legumes haulms (200-400 F CFA / kg) and the green fodder (50-82 F CFA / kg) in both cities. The activity is found profitable, making it possible to earn income of 67166 F CFA and 26335 F CFA per month respectively in Ouagadougou and Bobo-Dioulasso. The Fodders purchased are primarily used for sheep feeding. A better organization of the actors is necessary for the durability of the activity and the safeguarding of the environment.

(C) 2018 International Formulae Group. All rights reserved.

Keywords: Fodder, haulms, marketing, straw, urban breeding

\section{INTRODUCTION}

Au Burkina Faso, l'élevage occupe une frange importante de la population active et fournit des moyens d'existence à de nombreux ménages (Segni, 2008). En considérant les différents maillons du secteur, à savoir la production, la transformation et la commercialisation, on estime que le maillon «production » occupe annuellement une main d'œuvre totale d'environ 900000 personnes «équivalent temps plein», soit $7 \%$ environ de la population totale. Celui de la commercialisation et de la transformation entretiendraient $\quad 30 \quad 000$ personnes «équivalent temps plein» (IEPC, 2006). A ceux-ci, il faut ajouter les acteurs de l'approvisionnement en intrants et ceux des prestataires de service (vétérinaires, zootechniciens, acteurs privés ou publics de l'appui - conseil). Les vendeurs de fourrages pourraient également y contribuer, vue l'importance des spéculations sur les fourrages dans les centres urbains.

En effet, dans la but de satisfaire la demande en produits animaux de plus en plus croissante, face à la croissance démographique et à l'accroissement de l'urbanisation, on assiste à l'émergence de systèmes semi-intensifs à intensifs de production rencontrées en zones urbaines et périurbaines. Selon FAO (2012), 40\% des citadins en Afrique pratiquent une activité agricole, dont la production d'aliments de base, de légumes, de fruits, de lait, d'œufs, de viande et de poisson.

L'élevage en ville est sans doute une activité génératrice de revenu. Cependant, une contrainte majeure dans cette activité demeure l'alimentation des animaux, notamment le problème d'affouragement qui est couramment évoqué par les éleveurs (Sanon et al., 2014), et qui se pose de façon cruciale dans le milieu urbain où il y a un manque d'espace pour la pâture (Ali et al., 2003; Sanou et al., 2011).

D'où le développement d'un secteur d'activité constitué de vendeurs de fourrages de divers types, à travers les grands axes des centres urbains, dans le but de combler ce déficit fourrager, mais aussi de générer des revenus aux acteurs.

Cette étude vise à analyser la structure et le fonctionnement de la filière fourrage dans les deux grandes villes du Burkina Faso à savoir Ouagadougou et Bobo-Dioulasso, en vue de proposer des actions d'amélioration et de sauvegarde de l'environnement. De façon spécifique, il s'agit (i) d'inventorier et caractériser les sites de vente de fourrage dans ces deux villes, (ii) de caractériser les acteurs intervenant dans la vente de fourrage, (iii) d'identifier les types de fourrages faisant l'objet de vente, (iv) de déterminer les coûts en fonction de la nature du fourrage et (v) d'identifier les types d'acheteurs et ainsi les types d'animaux pour lesquels ces fourrages sont achetés. 


\section{MATERIEL ET METHODE}

\section{Sites d'étude}

L'étude s'est déroulée entre juin et août 2013, dans les deux grands centres urbains du Burkina Faso: Ouagadougou la capitale politique située au Centre du pays, et Bobo-Dioulasso la capitale économique, $2^{\mathrm{e}}$ ville du pays, située à l'Ouest.

La commune de Ouagadougou couvre une superficie de 37950 ha, située au centre de la province du Kadiogo dont elle est le chef-lieu, et aussi la capitale de la région. Cette ville enregistre le plus fort taux d'urbanisation du pays soit 77,5\%. Le climat est de type soudano-sahélienne, marqué par une pluviométrie moyenne annuelle de 800$900 \mathrm{~mm}$ de pluies, et une végétation dominée par la savane arbustive. La population est estimée à 1475223 habitants (INSD 2008). Dans la ville de Ouagadougou, certaines activités agro-sylvo-pastorales sont toujours pratiquées. Il s'agit de l'agriculture, de l'élevage, du maraîchage, de l'horticulture, l'arboriculture etc. Ces activités sont menées par beaucoup d'acteurs qui en tirent des revenus pour leur subsistance.

Bobo-Dioulasso est la seconde ville du pays, chef-lieu de la province du Houet, située à $365 \mathrm{~km}$ au sud-ouest de Ouagadougou. La ville appartient au domaine climatique Sud-Soudanien, caractérisé par des précipitations moyennes annuelles comprises entre 1100 et $1200 \mathrm{~mm}$. C'est le domaine des savanes de divers types: savanes arbustives, savanes arborées, savanes boisées, des forêts claires, des forêts galeries et des formations ripicoles. La population de la ville était estimée en 2006 à 489967 (INSD, 2008). L'agriculture urbaine existe également à Bobo-Dioulasso et joue pleinement son rôle de pourvoyeuse d'emploi et de revenus pour les populations (Robineau, 2013). Ces activités agricoles comprennent le maraîchage, la céréaliculture, l'horticulture, l'arboriculture, et l'élevage.

\section{Approche méthodologique}

L'étude a consisté dans un premier temps à recenser les différents sites de vente de fourrage dans les deux villes et estimer le nombre des acteurs présents. Puis, un échantillon d'environ $30 \%$ des vendeurs présents a été retenu pour les enquêtes en fonction de leur disponibilité. La méthode d'échantillonnage en boule de neige a été utilisée pour le choix des enquêtés par site. Elle a consisté à faire un choix aléatoire d'un premier vendeur qui est enquêté, puis solliciter pour inclure au moins cinq vendeurs dans l'enquête.

Des fiches d'enquête ont été élaborées comportant les informations suivantes: caractéristiques socio-économiques des vendeurs; les types de fourrages vendus en fonction des périodes de l'année et les coûts ; les modes d'approvisionnement en fourrage et les distances des sites de collecte; une estimation des quantités de fourrage vendus et des revenus de la vente des fourrages. Pour chaque type de fourrage, un échantillon de trois tas a été pesé pour déterminer le poids et rapporter le cout par $\mathrm{kg}$ de fourrage.

Au total, 85 vendeurs de fourrage ont été enquêtés dans les deux villes, dont 60 à Ouagadougou et 25 à Bobo-Dioulasso.

Des entretiens ont été également conduits au niveau des éleveurs urbains utilisateurs de ces fourrages à l'occasion des achats, en vue principalement de déterminer la destination des fourrages achetés.

\section{Analyse des données}

Les données ont été codifiées, saisies sur Excel et analysées avec le programme SPSS. Les statistiques descriptives (moyennes, pourcentage) ont été appliquées aux données collectées.

\section{RESULTATS \\ Caractéristiques des sites de vente Sites de Ouagadougou}

Une douzaine de sites de vente de fourrages a été recensée dans la ville de Ouagadougou. Plus de deux cents (200) personnes de tous âges et sexes confondus animent ces sites (Tableau 1). La durée d'implantation des sites est très variable, la plus récente date de 4 ans (Katr yaar) tandis que la plus ancienne existe depuis 27 ans 
(abattoir Ouaga), soit une moyenne de $11 \pm 6,7$ ans d'existence.

Il ressort qu'aucun des sites n'est aménagé ; seuls quelques hangars de fortune sont installés sur certains sites (Tanghin, Nioko) pour permettre aux vendeurs qui arrivent tôt de s'abriter du soleil et souvent de la pluie.

La plupart des sites sont permanents (75\%). La période de forte affluence sur les sites est la saison des pluies, période au cours de laquelle le fourrage vert existe en abondance. Cependant, en saison sèche, certains sites éprouvent souvent des moments de léthargie ; c'est le cas des sites du marché à bétail de Tanghin, de Cissin, et de Katr yaar, où la plus part des vendeurs se font livrer du foin et des sous-produits agricoles qui sont seulement disponibles à une certaine période de l'année. Le nombre d'acteurs dans les sites varie d'environ 10 vendeurs au marché de bétail de Cissin à plus de 40 vendeurs dans les sites de la zone 1 yaar et de Sapouy gare.

\section{Sites de Bobo-Dioulasso}

Dans la ville de Bobo-Dioulasso, les principaux sites de vente de fourrage recensés sont au nombre de 15 , avec 13 sites permanents et 2 sites plus ou moins saisonniers (Tableau 2).

La majorité des sites de vente de fourrage est localisée au niveau des axes routiers. Le nombre de vendeurs sur ces sites est estimé à environ 125 acteurs. Il n'existe pas de condition particulière pour l'exercice de cette activité de vente, ainsi que pour l'installation du vendeur sur un site donné, dans la mesure où l'installation n'est pas soumise à une autorisation préalable des autorités communales.

Les sites de ventes enquêtés ont une durée d'existence moyenne de $12 \pm 6$ ans. Le site le plus ancien date d'environ 30 ans (site de l'abattoir de Bobo) et le plus récent existe il y a seulement 4 ans. Les sites sont pour la plupart non aménagés (73\%). Là où il existe un aménagement, il s'agit plutôt d'abris sommaires, faits de bâches ou hangar en paille pour conserver les fourrages à l'abri des intempéries et des animaux en divagation. Les sites les plus importants dans la ville de Bobo-Dioulasso en termes d'acteurs présents sont ceux de Farakan (30 vendeurs), Route de Nasso (20 vendeurs), Lycée Cheick Anta Diop, (12 vendeurs) et Abattoir frigorifique (10 vendeurs).

\section{Caractéristiques socioéconomiques des vendeurs}

Le Tableau 3 montre les caractéristiques générales des vendeurs de fourrage dans les deux villes. La vente de fourrage est une activité principalement masculine. Environ $6 \%$ seulement des vendeurs sont de sexe féminin. Les vendeurs sont en majorité des adultes dans la tranche d'âge compris entre 20 et 50 ans (67\%). On note une proportion non négligeable des vendeurs de plus de 50 ans (28\%) dans la ville de Ouagadougou. Concernant le niveau d'instruction, l'enquête montre que la plupart des vendeurs de fourrage est non scolarisée avec une forte proportion dans la ville de Bobo-Dioulasso (83\%). De façon générale, la vente de fourrage n'est pas l'activité principale des enquêtés qui pratiquent majoritairement l'agriculture. La proportion de ceux qui font de la vente de fourrage leur activité principale varie de 20,8\% à Bobo à $37,7 \%$ à Ouagadougou. Des personnes exerçant d'autres types d'activités s'intéressent aussi à la vente de fourrage. Il s'agit des élèves, des artisans et des petits commerçants (environ $13 \%$ des enquêtés). Les élèves interviennent dans cette activité en saison des pluies qui coïncide avec la période des vacances scolaires et d'abondance de fourrage vert.

L'activité est récente pour $60 \%$ des vendeurs, même si certains l'exercent il y a plus de 5 ans (11\%). La majorité la pratique de façon permanente à Ouaga (69\%), tandis qu'à Bobo c'est la pratique de façon saisonnière qui domine $(71 \%)$.

\section{Fourrages commercialisés}

Circuit de commercialisation

Le circuit de commercialisation relie le préleveur/collecteur de fourrage, le vendeur et l'acheteur. Ce circuit est court 
puis que dans la plupart des cas, le préleveur/collecteur est en même temps le vendeur (91 et $83 \%$ respectivement à Ouaga et à Bobo). Seulement $8,8 \%$ des vendeurs à Ouaga et $16,7 \%$ à Bobo se font livrer le fourrage sur leur site de vente, par des agriculteurs pour les résidus de culture ou des élèves pour l'herbe verte en saison des pluies. La Figure 1 montre le circuit de commercialisation du fourrage dans les deux villes.

\section{Types de fourrages, sites de collecte et moyen de transport}

Les types de fourrages vendus sont très variés comprenant de l'herbe fraîche, du foin des pâturages, des résidus de cultures (fanes de légumineuses et pailles de céréales) et les fourrages ligneux (feuilles et/ou gousses d'arbres fourragers). L'herbe fraîche des pâturages naturels constitue le principal type de fourrage vendu par tous les vendeurs de la ville de Ouagadougou, suivi par le foin des herbacées $(77 \%)$ et les fanes de légumineuses $(72 \%)$.

Dans la ville de Bobo-Dioulasso, l'herbe fraîche est également le plus vendu par $83 \%$ des vendeurs; ensuite viennent les fanes de légumineuses (33\%) et le foin des herbacées $(20,83 \%)$ (Figure 2).

Les lieux de prélèvement des fourrages sont constitués par la brousse de façon générale, suivie par les champs et les jachères, puis les bas-fonds (Tableau 4). Les aires protégées sont aussi exploitées par certains vendeurs de fourrage. Ces aires protégées sont constituées de la forêt classée du parc Bangr Weogo à Ouagadougou et la forêt classée de Denderesso à BoboDioulasso. Pour la collecte du fourrage, les collecteurs parcourent des distances moyennes variant de 23,69 km (5 à $70 \mathrm{~km})$ à Ouagadougou et 18 km (7 à 30 km) à BoboDioulasso. Dans la ville de Ouagadougou, les vendeurs de fourrage estiment effectuer en moyenne 26 voyages par mois, tandis que ceux de Bobo-Dioulasso font en moyenne 12 voyages.

Les moyens utilisés pour le transport du fourrage des lieux de récolte aux points de vente sont de quatre types. Le vélo est le principal mode de transport utilisé par plus de $80 \%$ des vendeurs dans les deux villes. Les motos constituent le $2^{\mathrm{e}}$ moyen de transport utilisé par environ $15 \%$ des vendeurs à Ouagadougou et $21 \%$ environ à Bobo-Dioulasso. Le transport à pied n'est pas aussi négligeable surtout à Bobo-Dioulasso où environ $17 \%$ des vendeurs transportent le fourrage sur la tête, vers le lieu de vente. Les véhicules de transport en commun constituent le $4^{\mathrm{e}}$ moyen utilisé de façon occasionnelle.

\section{Commercialisation des fourrages}

Les fourrages sont vendus en tas ou fagot dont le poids varie suivant le type de fourrage et le volume de conditionnement. Le Tableau 5 montre le prix de vente moyen rapporté au kg par type de fourrage. De façon générale, le prix du fourrage est plus élevé dans les sites de Ouagadougou comparé aux sites de Bobo-Dioulasso. Les fanes de légumineuses ont le coût le plus élevé (200 à 400 F CFA / kg), suivi par l'herbe fraîche (50 à $82 \mathrm{~F} \mathrm{CFA} \mathrm{/} \mathrm{kg).} \mathrm{Les} \mathrm{pailles} \mathrm{de} \mathrm{céréales} \mathrm{sont}$ les moins chers (20 à 65 F CFA / kg).

Cette vente de fourrages permet aux acteurs d'engranger des revenus substantiels surtout dans la ville de Ouagadougou. Ainsi, la vente de fourrage rapporterait en moyen 26 335 F CFA par mois dans la ville de BoboDioulasso, contre $67166 \quad \mathrm{~F} \quad \mathrm{CFA}$ à Ouagadougou (Tableau 6). Les revenus de cette activité sont destinés principalement à couvrir les besoins de soins de santé, de scolarité des enfants et d'alimentation du ménage. Pour les vendeurs à BoboDioulasso, ces besoins sont d'égale importance tandis qu'à Ouagadougou, ce sont les soins de santé qui sont les plus évoqués suivis de la scolarité des enfants.

\section{Caractéristiques des acheteurs de fourrages}

Les éleveurs acheteurs de fourrage sont en majorité des hommes $(85,7 \%$ et $92,3 \%$ respectivement à Ouagadougou et Bobo-Dioulasso) avec un âge moyen de 45 ans et 43 ans dans les deux villes. Ils sont pour la plupart scolarisés: $52,3 \%$ à 
Ouagadougou et $69,3 \%$ à Bobo-Dioulasso ont fait au moins le niveau primaire. L'élevage n'est pas une activité principale pour ces acheteurs de fourrage; 95,6\% d'entre eux dans la zone Ouagadougou et $53,8 \%$ à Bobo-Dioulasso exercent des activités telles que le commerce, l'artisanat, ou sont fonctionnaires en activité ou à la retraite. Ils mènent cet élevage comme activité secondaire et la durée d'exercice est en moyenne de 6 ans à Ouagadougou et 10 ans à Bobo-Dioulasso. Les animaux alimentés sont élevés dans le système semiintensif $(100 \%$ des animaux à Ouagadougou et $61,5 \%$ à Bobo-Dioulasso). La taille moyenne du troupeau entretenu est de 5 et 9 animaux respectivement à Ouagadougou et à Bobo-Dioulasso.

Plusieurs espèces animales sont alimentées avec les fourrages achetés: ovins, bovins, caprins, équins et asins. L'alimentation des ovins vient en tête dans les deux villes avec $76 \%$ et $69 \%$ des acheteurs à Ouagadougou et à BoboDioulasso, suivis par celle des bovins (Tableau 7).

Tableau 1 : Caractéristiques des sites de vente de fourrages à Ouagadougou

\begin{tabular}{llllllc}
\hline $\begin{array}{l}\mathbf{N}^{\circ} \\
\text { ordre }\end{array}$ & Nom du site & Quartier & $\begin{array}{l}\text { Etat du } \\
\text { site }\end{array}$ & Fréquence & $\begin{array}{l}\text { Durée } \\
(\mathbf{a n})\end{array}$ & $\begin{array}{l}\text { Nombre } \\
\text { vendeurs }\end{array}$ \\
\hline 01 & Abattoir & Kossodo & $\begin{array}{l}\text { Non } \\
\text { Aménagé }\end{array}$ & Permanent & 27 & $10-20$ \\
\hline 02 & Bangr wéogo & Zone du Bois & $\begin{array}{l}\text { Non } \\
\text { Aménagé }\end{array}$ & Permanent & 13 & $10-20$ \\
\hline 03 & Ex-marché bétail & Gounghin & $\begin{array}{l}\text { Non } \\
\text { Aménagé }\end{array}$ & Temporaire & 18 & $10-20$ \\
\hline 04 & Katr yaar & Katr yaar & $\begin{array}{l}\text { Non } \\
\text { Aménagé }\end{array}$ & Temporaire & 4 & $10-20$ \\
\hline 05 & Marché à bétail & Cissin & $\begin{array}{l}\text { Non } \\
\text { Aménagé }\end{array}$ & Temporaire & 5 & 10 \\
\hline 06 & Nioko1 & Nioko1 & $\begin{array}{l}\text { Non } \\
\text { Aménagé }\end{array}$ & Permanent & 9 & $10-20$ \\
\hline 07 & Sapouy gare & Patte d'oie & $\begin{array}{l}\text { Non } \\
\text { Aménagé }\end{array}$ & Permanent & 10 & $>40$ \\
\hline 08 & Tambogdin yaar & Karpala & $\begin{array}{l}\text { Non } \\
\text { Aménagé }\end{array}$ & Permanent & 7 & $20-40$ \\
\hline 09 & Tampouy & Tampouy & $\begin{array}{l}\text { Non } \\
\text { Aménagé }\end{array}$ & Permanent & 7 & $10-20$ \\
\hline 10 & Tanghin & Tanghin & $\begin{array}{l}\text { Non } \\
\text { Aménagé }\end{array}$ & Permanent & 6 & $20-40$ \\
\hline 11 & Toéssin yaar & Tampouy & $\begin{array}{l}\text { Non } \\
\text { Aménagé }\end{array}$ & Permanent & 13 & $20-40$ \\
\hline 12 & Zone 1 yaar & Zone 1 & $\begin{array}{l}\text { Non } \\
\text { Aménagé }\end{array}$ & Permanent & 17 & $>40$ \\
\hline
\end{tabular}


Tableau 2: Sites de vente de fourrages à Bobo-Dioulasso

\begin{tabular}{|c|c|c|c|c|c|c|}
\hline $\begin{array}{l}\mathbf{N}^{\circ} \\
\text { ordre }\end{array}$ & Nom du site & Quartier & $\begin{array}{l}\text { Etat du } \\
\text { site }\end{array}$ & Fréquence & $\begin{array}{l}\text { Durée } \\
\text { (an) }\end{array}$ & $\begin{array}{l}\text { Nombre } \\
\text { vendeurs }\end{array}$ \\
\hline 01 & Route de Nasso & Colsama & $\begin{array}{l}\text { Non } \\
\text { Aménagé }\end{array}$ & Permanent & 10 & 20 \\
\hline $\mathbf{0 2}$ & Lycée Cheick Anta Diop & Secteur 22 & $\begin{array}{l}\text { Non } \\
\text { Aménagé }\end{array}$ & Permanent & 10 & 12 \\
\hline 03 & Pont d'Accart Ville & Accart Ville & $\begin{array}{l}\text { Non } \\
\text { Aménagé }\end{array}$ & Permanent & 10 & 5 \\
\hline 04 & Farakan & Farakan & $\begin{array}{l}\text { Non } \\
\text { Aménagé }\end{array}$ & Permanent & $15-20$ & 30 \\
\hline 05 & Bindougousso & Bindougousso & Bâches & Permanent & 10 & 8 \\
\hline 06 & Nieneta & Nieneta & $\begin{array}{l}\text { Non } \\
\text { Aménagé }\end{array}$ & Permanent & 10 & 6 \\
\hline 07 & Route du Mali 1 & Yégueré & $\begin{array}{l}\text { Non } \\
\text { Aménagé }\end{array}$ & Permanent & 6 & 3 \\
\hline 08 & Route du Mali 2 & Colma & Bâche & Permanent & 6 & 4 \\
\hline 09 & Marché de bétail & Colma & $\begin{array}{l}\text { Non } \\
\text { Aménagé }\end{array}$ & Permanent & 6 & 4 \\
\hline 10 & Grand Marché & Hamdalaye & Bâche & Permanent & 20 & 4 \\
\hline 11 & Saint Etienne & Saint Etienne & $\begin{array}{l}\text { Non } \\
\text { Aménagé }\end{array}$ & Temporaire & 15 & 2 \\
\hline 12 & Aéroport de Bobo & Secteur 21 & $\begin{array}{l}\text { Non } \\
\text { Aménagé }\end{array}$ & Permanent & 5 & 3 \\
\hline 13 & $\begin{array}{l}\text { Lycée Mixte d'Accart- } \\
\text { ville }\end{array}$ & Accart-ville & $\begin{array}{l}\text { Non } \\
\text { Aménagé }\end{array}$ & Temporaire & 4 & 2 \\
\hline 14 & Marché des fruits & Secteur 21 & Bâches & Permanent & 20 & 8 \\
\hline 15 & $\begin{array}{l}\text { Abattoir frigorifique ( } 4 \\
\text { sous sites) }\end{array}$ & Niénéta & $\begin{array}{l}\text { Hangar et } \\
\text { Bâches }\end{array}$ & Permanent & 30 & 10 \\
\hline
\end{tabular}

Tableau 3: Caractéristiques socioéconomiques des vendeurs de fourrage ( $\%$ des répondants).

\begin{tabular}{lllll}
\hline & & Ouagadougou & $\begin{array}{l}\text { Bobo- } \\
\text { Dioulasso }\end{array}$ & Général \\
\hline Sexe & Homme & 93,40 & 95,80 & 94,60 \\
& Femme & 6,60 & 4,20 & 5,40 \\
\hline Age & $<20$ ans & 4,90 & 16,70 & 10,80 \\
& $20-50$ & 67,20 & 66,66 & 66,93 \\
& $>50$ ans & 27,90 & 16,70 & 22,30 \\
\hline Niveau d'instruction & Non Scolarisé & 59,00 & 83,30 & 71,15 \\
& Alphabétisé & 1,60 & 8,30 & 4,95 \\
& Primaire & 31,10 & 4,20 & 17,65 \\
& Secondaire & 8,20 & 4,20 & 6,20 \\
\hline Activité principale & Agriculture/Elevag & 49,10 & 66,70 & 57,90 \\
& e & 37,70 & 20,80 & 29,25 \\
\hline
\end{tabular}




\begin{tabular}{lllll}
\hline & Vente de fourrage & 13,10 & 12,50 & 12,80 \\
& Autres & & & \\
\hline Ancienneté dans l'activité (an) & $<2$ & 60,70 & 58,30 & 59,5 \\
& $2-5$ & 27,90 & 29,20 & 28,55 \\
& $>5$ & 11,50 & 12,50 & 12 \\
\hline Fréquence de l'activité & Permanent & 68,90 & 29,20 & 49,05 \\
& Saisonnier & 31,10 & 70,80 & 50,95 \\
\hline Taille de ménage & & 5,31 & 5,33 & 5,32 \\
\hline
\end{tabular}

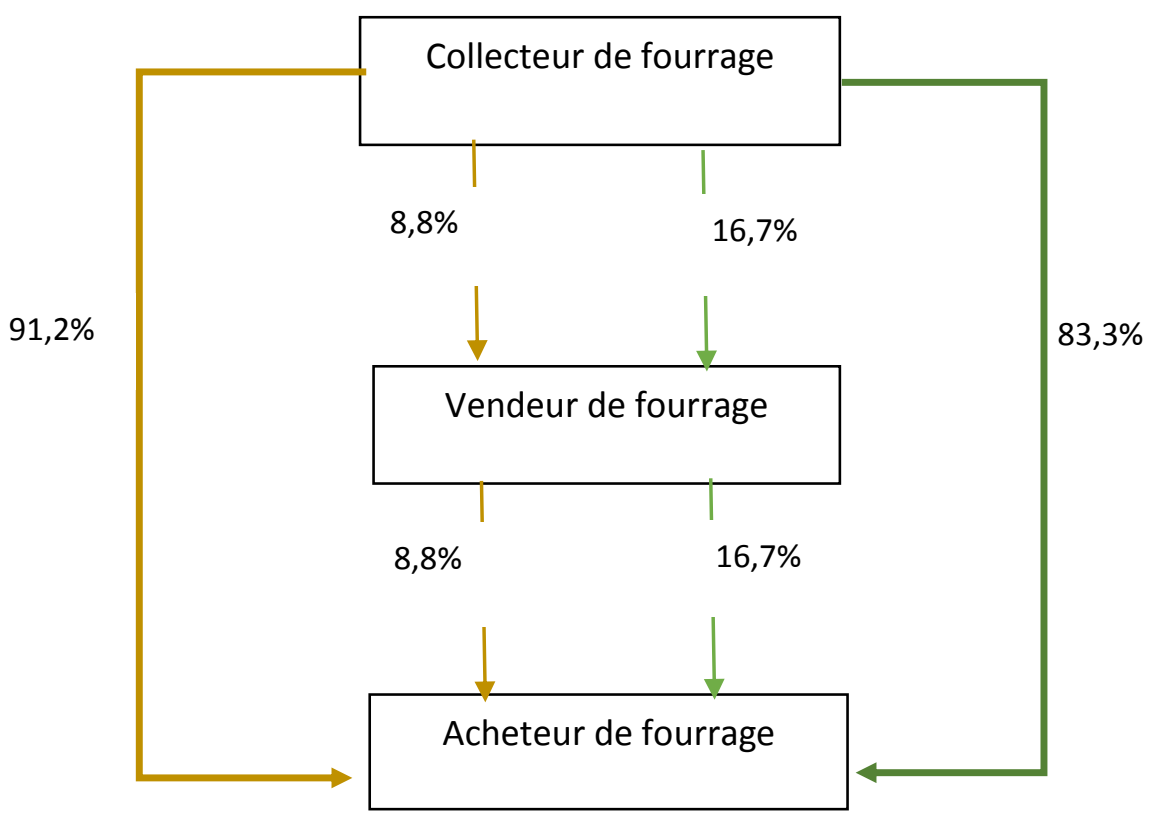

Figure 1: Circuit de commercialisation du fourrage à Ouagadougou (couleur marron) et à BoboDioulasso (couleur verte).

Tableau 4: Sites de collecte de fourrages et moyens de transport (\% des répondants).

\begin{tabular}{lllll}
\hline & & Ouagadougou & Bobo-Dioulasso & Générale \\
\hline \multirow{4}{*}{ Sites de collecte } & Brousse & 88,52 & 95,83 & 92,17 \\
& Aires protégées & 6,56 & 4,17 & 5,36 \\
& Champs \& jachères & 73,77 & 50,00 & 61,88 \\
& Basfonds & 73,77 & 29,17 & 51,47 \\
\hline \multirow{4}{*}{ Moyen de transport } & Pied & 8,20 & 16,67 & 12,43 \\
& Vélo & 85,25 & 83,33 & 84,29 \\
& Moto & 14,75 & 20,83 & 17,79 \\
& Véhicule & 1,64 & 4,17 & 2,90 \\
\hline \multicolumn{2}{l}{ Nombre de voyage /mois } & 26 & 12 & 19 \\
\hline Distance moyenne parcourue & 23,69 & 18,46 & 21,07 \\
\hline
\end{tabular}




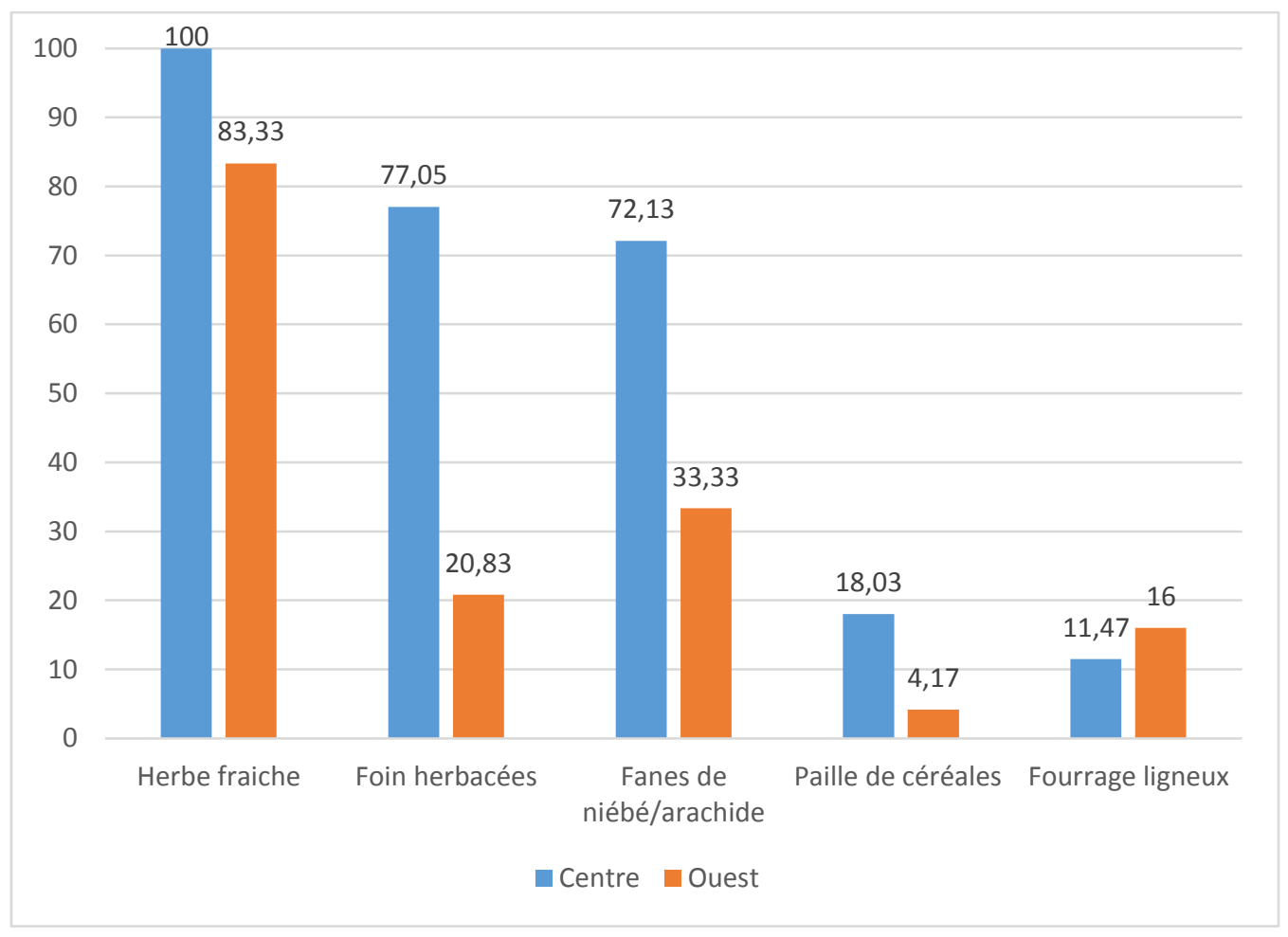

Figure 2: Types de fourrages vendus dans les deux villes.

Tableau 5: Prix de vente des fourrages (en F CFA/kg).

\begin{tabular}{lll}
\hline Type fourrage & Ouagadougou & Bobo-Dioulasso \\
\hline Herbe fraîche & 82 & 50 \\
\hline Foin & 91 & 35 \\
\hline Paille céréale & 65 & 20 \\
\hline Fanes de Légumineuses & 260 & $200-400$ \\
\hline
\end{tabular}

Tableau 6: Revenu de la vente des fourrages et destination (\% des répondants).

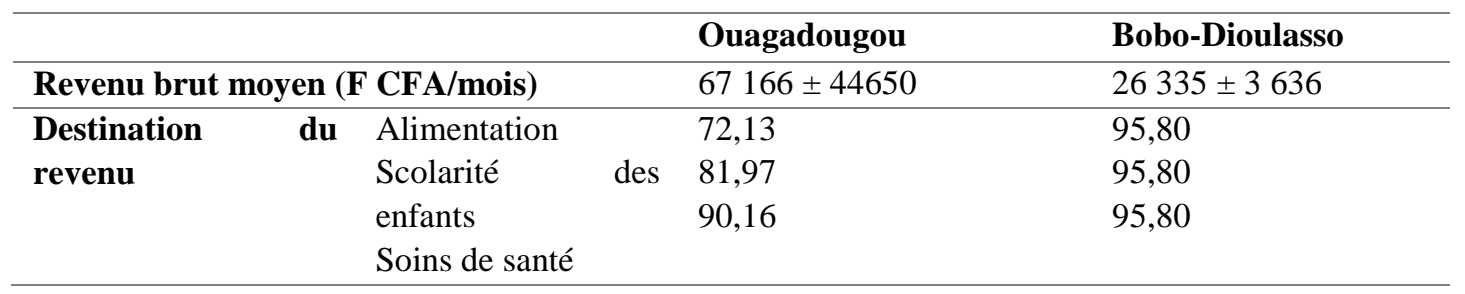


Tableau 7: Profil des éleveurs - acheteurs de fourrage.

\begin{tabular}{|c|c|c|c|c|}
\hline & & Ouagadougou & Bobo-Dioulasso & Général \\
\hline \multirow[t]{2}{*}{ Sexe $(\%)$} & Homme & 85,70 & 92,30 & 89 \\
\hline & Femme & 14,30 & 7,70 & 11 \\
\hline \multicolumn{2}{|l|}{ Age moyen (ans) } & $45 \pm 10,2$ & $43 \pm 14,7$ & 44 \\
\hline \multirow{4}{*}{$\begin{array}{l}\text { Niveau d'instruction } \\
(\%)\end{array}$} & Non Scolarisé & 38,10 & 23,10 & 30,60 \\
\hline & Alphabétisé & 9,50 & 7,70 & 8,60 \\
\hline & Primaire & 28,60 & 30,80 & 29,70 \\
\hline & Secondaire & 23,80 & 38,50 & 31,15 \\
\hline \multirow{3}{*}{$\begin{array}{l}\text { Activité } \\
(\%)\end{array}$} & Agriculture & 4,80 & 23,10 & 13,95 \\
\hline & Elevage & - & 23,10 & 11,55 \\
\hline & Autres activités* & 95,60 & 53,80 & 74,70 \\
\hline \multirow[t]{2}{*}{ Type d'élevage (\%) } & Intensif & - & 38,50 & 19,25 \\
\hline & Semi-intensif & 100 & 61,50 & 80,75 \\
\hline \multicolumn{2}{|c|}{ Ancienneté dans l'activité (an) } & $6,6 \pm 5,9$ & $10 \pm 6,5$ & 8,3 \\
\hline \multicolumn{2}{|c|}{ Taille du troupeau } & $5 \pm 3$ & $9 \pm 6$ & 7 \\
\hline \multirow{5}{*}{ Espèce animale (\%) } & Ovins & 76,19 & 69,23 & 72,71 \\
\hline & Bovins & 19,05 & 46,15 & 32,60 \\
\hline & Caprins & 4,76 & 15,38 & 10,07 \\
\hline & Equins & 9,52 & 7,69 & 8,60 \\
\hline & Asins & 4,76 & 15,38 & 10,07 \\
\hline
\end{tabular}

* : commerce, artisanat, fonctionnaire.

\section{DISCUSSION}

\section{Sites de vente}

Le nombre important de sites inventorié dans les deux villes démontre l'importance de l'activité de vente de fourrages. Le résultat obtenu dans la ville de Bobo-Dioulasso est similaire à celui rapporté Sanou et al. (2016) qui ont noté 15 sites permanents, auxquels s'ajoutent 6 sites saisonniers. Dans notre étude, le recensement a concerné surtout les principaux sites et l'existence d'autres sites de moins grande envergure a été signalée.

La durée d'implantation des sites les plus anciens (30 ans à l'abattoir frigorifique de Bobo-Dioulasso et 27 ans à l'abattoir frigorifique à Kossodo, Ouagadougou) montre le début probable de cette activité de commercialisation des fourrages, qui a certainement démarré dans les abords des marchés à bétail avant de s'étendre dans les différentes artères des villes. D'ailleurs, les sites sont implantés aux abords des grands axes et marchés de bétail, ce qui facilite l'accès aux acheteurs.

La plupart des sites est permanent, cependant le manque d'aménagement dans les sites présage une certaine précarité de l'activité, dans la mesure où les vendeurs peuvent être déguerpis à tout moment si le besoin d'occupation de l'espace se fait sentir par les autorités.

\section{Caractéristiques des vendeurs}

La vente de fourrage est une activité essentiellement masculine. On note une faible implication des femmes dans la vente de fourrage, qui pourrait s'expliquer d'une part par la pénibilité de la collecte du fourrage, et d'autre part le fait qu'elles s'intéressent plus à la vente d'autres types d'aliments du bétail. Ainsi, Soubeiga (2014) a noté une proportion importante de femmes (environ 40\%) dans la vente d'aliments concentrés constitués de sons locaux collectés auprès des meuniers, de drèches de bière local (dolo) et tourteaux de coton dans la ville de Ouagadougou. Maman (2014) a aussi noté une faible proportion de femmes dans la commercialisation du fourrage à Niamey comparativement aux hommes qui constituaient $97,67 \%$ de la population de vendeurs. Cet auteur rapporte également que la commercialisation du fourrage est une activité secondaire pour la 
majorité des vendeurs (62.8\% contre 37,2\% qui la pratique comme seule activité). Ce qui corrobore nos résultats dans la ville de Ouagadougou où $37,7 \%$ des vendeurs pratiquent cette activité de façon régulière.

Le nombre important des vendeurs de la zone de Ouagadougou, qui pratiquent cette activité de façon permanente (69\%), indiquerait une certaine rentabilité permettant aux acteurs de satisfaire leurs besoins quotidiens.

\section{Fourrage vendu}

Les fourrages vendus proviennent de plusieurs zones de prélèvement (fourrage naturel) et de production (résidus de culture). La faible importance des résidus de cultures dans la ville de Bobo-Dioulasso comparée à Ouagadougou pourrait s'expliquer par l'abondance des fourrages naturels dans la zone sud-soudanienne par rapport à celle nord-soudanienne où se situe Ouagadougou.

Le lieu privilégié de prélèvement étant la brousse dans les deux sites, suppose une pression de plus en plus forte sur ces milieux, dans la mesure où d'autres types d'exploitation sont inhérentes à ces milieux, notamment la pâture des animaux et la fauche $\mathrm{du}$ fourrage par les éleveurs pour la conservation et utilisation à des périodes de pénurie alimentaire du bétail. Ces actions auront pour conséquence la rareté des espèces les plus appréciées, la dégradation des zones de prélèvement et une perte de la biodiversité à long terme. En effet, la coupe au stade de floraison ou épiaison des plantes annuelles, stade végétatif recommandé pour avoir le fourrage de bonne qualité, réduit la densité des plantes des espèces concernées, avec pour conséquence leur rareté, voire leur disparition à long terme dans le pâturage. Des mesures d'accompagnement s'avèrent alors nécessaire pour la sauvegarde de l'environnement et la pérennité de l'activité de vente de fourrage. La distance parcourue par les préleveurs allant jusqu'à $40 \mathrm{~km}$ pour s'approvisionner en fourrage, témoigne de la faible disponibilité des ressources dans l'environnement immédiat des centres urbains et/ou de la multiplicité des acteurs intervenant dans l'activité. De même, l'exportation des résidus de cultures pour la vente pourrait exposer les sols cultivés à l'érosion et réduire leur productivité si aucune mesure d'accompagnement n'est prise pour restaurer ces sols (Kouelo et al., 2017).

La pratique des cultures fourragères pourraient contribuer à augmenter la disponibilité de fourrage de bonne qualité, tout en minimisant les risques de dégradation des pâturages. Cependant, force est de reconnaitre que les vendeurs ne sont pas très ouverts à cette technologie, évoquant le manque et la difficulté d'accès à la terre pour cette culture (communication personnelle). Cette activité pourrait être pratiquée, soit par des préleveurs sur de petites superficies avec des espèces améliorées à forte productivité, ou par des agriculteurs qui approvisionnent les vendeurs. Pour cela, les cultures fourragères à double objectifs pourraient être privilégiées. $\mathrm{Ce}$ qui constituerait un réel facteur d'intégration agriculture - élevage, comme rapporté par Obulbiga et al. (2015). D'ailleurs, la rentabilité de la vente des fanes de légumineuses par rapport aux autres types de fourrage témoigne de bonnes perspectives. Le cout élevé des fanes de légumineuses est sans doute en rapport avec leur valeur nutritive élevée comparée aux autres types de fourrage. Avec un prix de vente moyen d'environ $300 \mathrm{~F}$ CFA $/ \mathrm{kg}$ de fanes de légumineuses, ces fourrages reviennent plus chers que les aliments concentrés, tels que les tourteaux de coton dont la tonne fait 150000 FCFA, soit $150 \mathrm{~F} \mathrm{CFA} \mathrm{/} \mathrm{kg.}$

Ainsi, cette activité génère des revenus plus ou moins importants pour les acteurs. Le montant de 67166 FCFA enregistré dans la zone de Ouagadougou est supérieur au SMIG au Burkina Faso qui est de l'ordre $32218 \mathrm{~F}$ CFA (en 2010). Cette valeur est également supérieure à celle rapportée par Sanou et al. (2011) qui ont relevé un produit brut annuel moyen de $675000 \mathrm{~F}$ CFA pour l'ensemble des vendeurs, soit $56250 \mathrm{~F}$ CFA par mois dans la ville de Bobo-Dioulasso. Nos résultats dans cette ville sont cependant inférieurs à ceux rapportés par ces auteurs (26 $500 \mathrm{~F}$ $\mathrm{CFA} /$ mois en moyenne). Ils sont cependant plus élevés que la moyenne de 14943 F CFA rapporté par Kiema et al. (2012) pour la vente de fourrage naturel en zone sahélienne du Burkina Faso. Ces fourrages constituent, de ce 
fait, des sources importantes d'activités génératrices de revenu, contribuant ainsi à la résorption du chômage. Aboh (1999) avait également rapporté l'importance de la commercialisation du fourrage comme activité génératrice de revenu au Sud Benin avec des revenus supérieurs au SMIG. De même, Maman (2014) a souligné la rentabilité financière du commerce de fourrages à Niamey, et particulièrement celle des légumineuses fourragères, qui est plus élevée que pour les fourrages naturels.

\section{Caractéristiques des acheteurs / éleveurs}

Les caractéristiques des éleveurs urbains sont similaires à ceux reportés par Tegegne et al. (2003) à Addis-Abeba pour ce qui concerne le niveau d'éducation. En effet, ces auteurs ont noté que $70 \%$ des éleveurs urbains étaient scolarisés, d'âge supérieur à 30 ans pour la plupart et menant cette activité de façon secondaire.

Les ovins constituant la première espèce pour laquelle les fourrages sont achetés, suppose une prédominance de cette espèce dans les élevages urbains. Ce qui concorde avec les observations de Maman Lawal et al. (2018), qui ont noté une prédominance des ovins dans le milieu urbain de Niamey. La taille relativement modeste des troupeaux pour lesquels les fourrages sont achetés fait penser à un rôle d'épargne et/ou social de cette activité. En effet, il s'agit des acteurs disposant de moyens assez modestes avec peu d'investissement dans l'habitat, la plupart laissant les animaux sous un hangar dans la cour familiale. Cependant, l'activité contribue à la satisfaction des besoins des acteurs et à l'approvisionnement de la ville en produits animaux. Néanmoins, des contraintes existent notamment en saison sèche où les fourrages sont rares et de coûts élevés.

\section{Conclusion}

Cette étude nous a permis de comprendre le secteur de la vente du fourrage, les acteurs impliqués ainsi que le fonctionnement de la filière. On retient que l'activité est rentable et implique beaucoup d'acteurs leur permettant de subvenir à leurs besoins. Cependant, le secteur n'est pas organisé, ce qui présage sa précarité, même si son importance dans le soutien alimentaire des élevages urbains est fondamentale.

Cette commercialisation basée essentiellement sur l'exploitation des ressources naturelles a des conséquences à long termes sur ces ressources. D'où la nécessité d'une meilleure organisation des acteurs et surtout leur sensibilisation à la pratique de cultures fourragères pour la sauvegarde de l'environnement et la durabilité de leur activité. Il s'avère nécessaire de prendre en compte ces acteurs dans les filières de productions animales, vu leur nombre de plus en plus important et la place que l'activité occupe dans la génération de revenus en milieu urbain.

\section{CONFLIT D'INTERETS}

Les auteurs déclarent ne pas avoir de conflits d'intérêts en relation avec cet article.

\section{CONTRIBUTIONS DES AUTEURS}

HOS a validé le protocole d'étude, a été responsable de l'exploitation des résultats et de la rédaction du manuscrit. SSS et MFO ont contribué à l'élaboration des fiches d'enquêtes et à l'analyse des données. FAO et IB ont contribué à la collecte et au dépouillement des données d'enquête. Tous les auteurs ont pris connaissance du manuscrit rédigé.

\section{REFERENCES}

ABOH AB. 1999. La commercialisation de fourrages verts au marché de Zongo à Cotonou: état des lieux et contraintes. Bulletin de la Recherche Agronomique, $25: 1-8$.

Ali L, Van den Bossche P, Thys E. 2003. Enjeux et contraintes de l'élevage urbain et périurbain des petits ruminants à Maradi au Niger : quel avenir ? Revue Élev. Méd. Vét. Pays Trop., 2003, 56 (12) 73-82. DOI: https://doi.org/10.19182/remvt.987 9

FAO. 2012. Pour des villes plus vertes en Afrique. Premier rapport d'étape sur l'horticulture urbaine et périurbaine. Food and Agriculture Organization of the United Nations, Rome, 111p. 
IEPC (Initiative Elevage Pauvreté et Croissance). 2006. Proposition pour un document national. Rapport principal ; version provisoire ; $135 \mathrm{p}$.

INSD. 2008. Recensement général de la population et l'habitat de 2006. Résultats définitifs. Ouagadougou, Burkina Faso ; $52 \mathrm{p}$.

KIEMA André, SAWADOGO Issa, OUÉDRAOGO Tinrmegson et NIANOGO Aimé Joseph, 2012. Stratégies d'exploitation du fourrage par les éleveurs de la zone sahélienne du Burkina Faso. Int. J. Biol. Chem. Sci., 6(4): 1492-1505. DOI : http://dx.doi.org/10.4314/ijbcs.v6i4.8

Kouelo AF, Houngnandan P, Azontonde A, Benmansour M, Bekou J, Akplo T. 2017. Effet des pratiques de conservation du sol sur la croissance et les composantes du rendement du maïs dans le bassin versant de Lokogba au Bénin. Agronomie Africaine, 29(1) : 65 - 78 .

Maman S. 2014. Analyse du système de commercialisation du Fourrage dans la ville de Niamey (Niger). Mémoire de Master: Productions Animales et Développement Durable. Ecole Interétats des Sciences et Médecine vétérinaires (EISMV) de Dakar, Sénégal, 40p.

Maman Lawal AA, Chaibou M, Mani M, Garba MM, Gouro AS. 2018. Pratiques d'éleveurs et résultats économiques d'élevage dans les exploitations urbaines et périurbaines de Niamey. Int. J. Biol. Chem. Sci., 12(1): 294-309. DOI : https://dx.doi.org/10.4314/ijbcs.v12i1.24

Robineau O. 2013. Vivre de l'agriculture dans la ville africaine. Une géographie des arrangements entre acteurs à BoboDioulasso, Burkina Faso. Thèse de doctorat, Géographie et Aménagement de l'Espace. Université Paul Valéry Montpellier III, France ; 379p.

Obulbiga MF, Bougouma V, Sanon HO. 2015. Amélioration de l'offre fourragère par l'association culturale céréale- légumineuse à double usage en zone nord soudanienne du Burkina Faso. Int. J. Biol. Chem. Sci., 9(3): 1431-1439. DOI: http://dx.doi.org/10.4314/ijbcs.v9i3.26

Sanon Hadja Oumou, Drabo Aminata, Sangare Mamadou, Kiendrebeogo Timbilfou, Gomgnibou Alain. 2014. Caractérisation des pratiques d'embouche bovine dans l'Ouest du Burkina Faso. Int. J. Biol. Chem. Sci., 8(2): $\quad 536-550 . \quad$ DOI: http://dx.doi.org/10.4314/ijbcs.v8i2.13

Sanou KF, Nacro S, Ouédraogo M, Ouédraogo S, Kaboré-Zoungrana C. 2011. La commercialisation de fourrages en zone urbaine de Bobo-Dioulasso (Burkina Faso) : pratiques marchandes et rentabilité économique. Cah Agric, 20: 487-93.

DOI: https://doi.org/10.1684/agr.2011.05 30

Sanou KF, Ouédraogo S, Nacro S, Ouédraogo M, Kaboré-Zoungrana C. 2016. Durabilité de l'offre et valeur nutritive des fourrages commercialisés en zone urbaine de Bobo-Dioulasso, Burkina Faso. Cah. Agric., 25, 15002 ; 10p. DOI: 10.1051/cagri/2016007

Soubeiga LM. 2014. Analyse des aliments du bétail vendus dans la ville de Ouagadougou : Structure et fonctionnement des marchés. Rapport de stage de fin de cycle de techniciens supérieurs d'élevage. ENESA, Ouagadougou, Burkina Faso, 40p.

Tegegne A, Tadesse M, Alemayehu M, Woltedji D, Sileshi Z. 2003. Scoping study on peri-urban and urban livestock production in Addis Ababa, Ethiopia. In Urban livestock keeping in sub-Saharan Africa, Richards JI, Godfrey SH (Eds). Report of a workshop held on 3-5 March 2003 in Nairobi, Kenya. Natural Resources International Ltd, Aylesford, Kent, UK. ISBN: 0-95329274-9-0. 